\title{
Clinical \& etiological profile of acute undifferentiated fever in patients admitted to a teaching hospital in Pondicherry
}

\author{
Uthaya Sankar M.K. ${ }^{1}$, Perumbadi S. L. ${ }^{2}$, Shenbagaram K. ${ }^{3}$, Hanushraj R. ${ }^{4}$ \\ ${ }^{1}$ Dr. M. K. Uthaya Sankar, Professor, ${ }^{2}$ Dr. Suhaim Latif Perumbadi, Resident, ${ }^{3}$ Dr. Shenbagaram K., Assistant Professor, \\ ${ }^{4}$ Dr. Hanushraj R., Senior Resident, all authors are affiliated with Department of General Medicine, Sri Manakula \\ Vinayagar Medical College \& Hospital, Kalitheerthalkuppam, Madagadipet, Puducherry, 605107, India.
}

Corresponding Author: Dr. Suhaim Latif Perumbadi, Resident, Department of General Medicine, Sri Manakula Vinayagar Medical College \& Hospital, Kalitheerthalkuppam, Pondicherry. Email: suhaim.perumbadi@gmail.com

\begin{abstract}
Objectives: To identify the infectious aetiology and clinical features of Acute Undifferentiated Fever among patients admitted to a teaching hospital in Pondicherry. Design: Prospective observational study. Subjects: 270 cases comprising all adult patients ( $\geq 18$ years) admitted to the hospital with fever of less than 14 days duration, temperature $\geq 38^{\circ} \mathrm{C}$ upon admission and non-detection of any specific foci of infection by history, physical examination and routine investigations. Previously diagnosed cases of known fevers, collagen vascular disorders, endocrine disorders, malignancies, immunodeficient states, fever of duration $\geq 14$ days and drug induced fevers were excluded. Methods: All patients were examined and investigated according to study protocol after an informed consent to reach final diagnosis. Results: $38.1 \%$ cases were of Scrub Typhus aetiology, 20\% cases tested positive for dengue, $14.1 \%$ cases came positive for Enteric fever and Malaria was found in 5.6\% cases, all of $P$. vivax parasite. There was 1 case of TB, 1 case of candida infection and 1 case of Klebsiella pneumonia. 1 case tested positive for leptospirosis. Blood pathogens isolated were Pseudomonas and Enterococci with 1 case each. UTI was a frequent diagnosis with 7 cases of E. coli, 1 case of Enterococci and 1 case of Staphylococci. 1 case of HIV was detected. Conclusion: Scrub typhus was the most common aetiology of Acute Undifferentiated Fever followed by Dengue and Enteric fever.
\end{abstract}

Key words: Acute Undifferentiated Fever, Dengue, Enteric fever, Malaria, Scrub typhus

\section{Introduction}

Acute undifferentiated fever (AUF) is a common cause of patients seeking healthcare in India [1,2]. Unlike fever of unknown origin (FUO), which has a standard definition, AUF, also known as "acute febrile illness", "short febrile illness", or "acute fever" lacks an international consensus definition. Since FUO requires duration of fever to be longer than three weeks, some authors have defined AUF as fever that resolves within three weeks. Thus the term AUF is used to denote fevers that typically do not extend beyond 21 days, and lack localizable or organ-specific clinical features [3].

AUF poses a diagnostic and therapeutic challenge to the healthcare workers, particularly in limited resource settings. A number of viruses, bacteria, protozoa and rickettsia can cause AUF. The non-specificity of symptoms and signs and lack of availability of accurate

Manuscript received: $25^{\text {th }}$ May 2018

Reviewed: $4^{\text {th }}$ June 2018

Author Corrected: $10^{\text {th }}$ June 2018

Accepted for Publication: $16^{\text {th }}$ June 2018 diagnostics not only challenge the diagnosis but often leads to irrational use of antibiotics and antimalarials. Some fever syndromes have a more clear localization to skin and soft tissue, meninges or neural tissue, respiratory tract, or urinary tract. These syndromes have better developed guidelines for their management. On the other hand, AUF-syndromes have overlapping aetiologies, which makes their diagnosis and management challenging [4].

Those AUFs which persist, and total duration of illness becomes longer than three weeks are classified as FUO. Diagnosis of many aetiologies of AUF in the tropics can be established with help of simple tests, such as peripheral smear examination or rapid diagnostic tests (RDTs) for malaria or dengue [3, 5, 6]. Some other aetiologies need more sophisticated tests such as ELISA for rickettsial infections, MAT or ELISA for leptospirosis or PCR based tests for paramyxoviruses $[7,8,9]$. Depending on the nature of available 


\section{Original Research Article}

laboratory support, between a quarter and half of AUFs may remain undiagnosed and hence a better understanding of the prevalence and causes of AUF geographically would help in narrowing down the vast diagnostic workup needed to pin point the aetiological agent and guide to an optimal treatment to avoid unwanted usage of antibiotics and antimalarials [5, 10]. We study here the most common aetiologies and clinical features of AUF in a teaching hospital with patients hailing mostly from Rural Pondicherry and, Villupuram, Cuddalore and Thiruvannamalai districts of Tamil Nadu.

\section{Materials and Methodology}

The present study emphasizes on the infective aetiologies of Acute Undifferentiated fevers with special emphasis on clinical and demographic features among patients admitted in a teaching hospital in Pondicherry. This study was undertaken in the Dept. of General Medicine and required cases were taken from among the inpatients of the same during the period of November 2015 to May 2017.

Study design: Hospital based prospective observational study

Sample size: $270(\sim 15$ admissions per month multiplied by 18 months study period)

\section{Sample subjects:}

Inclusion criteria: All adult patients ( $\geq 18$ years) admitted to the hospital with fever of less than 21 days duration, with temperature $\geq 38^{\circ} \mathrm{C}$ upon admission and non-detection of any specific foci of infection by history, physical examination and routine investigations.

\section{Exclusion criteria}

- Previously diagnosed cases of,

- Known fevers

- Collagen vascular disorders

- Endocrine disorders

- Malignancies

- Immunodeficient states

- Fever of duration $\geq 21$ days

- Drug induced fever
Data entry and analysis: The data was entered in Microsoft Excel and analysed using Epi Data analysis V2.2.2.186 and STATA 12.0 software. The continuous variables like age, duration of fever and ESR at 1 hour were reported as Mean (SD) or median (Inter Quartile Range) based on distribution of data. The categorical variables such as gender, presence of symptoms (myalgia, jaundice, haemorrhage, conjunctival congestion, diarrhoea and cough or dyspnoea), test results (malaria slide examination, RDT for malaria, thyroid function test, sputum examination, dengue, scrub typhus, leptospirosis, Widal test, blood culture, urine culture, $\mathrm{HIV}, \mathrm{HCV}, \mathrm{Hbs} \mathrm{Ag}$ ), finding from chest $\mathrm{X}$ ray, USG findings and final diagnosis of undifferentiated fever were reported as proportions. In comparison analysis, the final diagnosis categories with less number of patients were excluded from analysis.

The association between continuous variables [age, duration of fever and ESR at 1 hour] and the final diagnosis of undifferentiated fever were assessed using one way ANOVA or Kruskal Wallis test and the association between categorical variable and the final diagnosis of undifferentiated fever were assessed using Chi Square test or Fisher's exact test based the cell values. The $\mathrm{p}$ value of $<0.05$ was considered for statistical significance.

Ethical consideration: The ethical approval for the study was obtained from the Institute Ethical committee of Sri Manakula Vinayagar Medical College and Hospital, Pondicherry. All ethical principles were adhered in the study

Data collection: All participants were briefed about the study in the language they comprehend and their willingness to participate in the study was obtained through informed consent form. Detailed clinical history was obtained from the selected patients and a thorough physical examination done for each. Blood, urine and sputum samples were taken from the study group and sent for the following investigations accordingly:

Investigations and methodology followed: Investigations were done as per the clinical assessment.

\section{Result}

Out of the 270 cases of patients of the age 18 and above, the mean age was 39.1 years. 53.7\% (145) were males and $46.3 \%$ (125) were females.

The mean duration of fever at presentation to clinic was of 6.9 days. $71.5 \%$ (193) of the patients complained of myalgia.10.4\% (28) of the patients with AUF in our study presented with diarrhoea. 39.6\% (107) of the patients in our 


\section{Original Research Article}

study presented with cough and/or dyspnoea. The mean ESR value among the study group after 1 hour reading was 33.4 mm. 5.6\% (15) cases of AUF came positive for P. vivax malaria by smear examination and 5.2\% (14) cases where also positive by Rapid Diagnostic Test. There were no other malarial parasites found in the study population. All cases suspected of a thyroid disorder were of euthyroid state among the 270 cases of AUF in our study group. 1 case was positive for Acid fast bacilli, 1 for Candida species and 1 for Klebsiella pneumonia among the 270 cases in the study group of AUF. Dengue was found to be positive among $20 \%$ (54) of the study population. Scrub typhus was found positive in $38.1 \%$ (103) of the study population. Widal test was positive for $14.1 \%$ (38) patients out of the study population.

Table-1: Final diagnosis of AUF.

\begin{tabular}{|c|c|c|}
\hline Final aetiology & Number & Percentage \\
\hline Dengue & 43 & 15.9 \\
\hline Dengue/Scrub typhus & 3 & 1.1 \\
\hline Enteric fever & 33 & 12.3 \\
\hline P.vivax malaria & 14 & 3.2 \\
\hline Scrub typhus & 103 & 38.1 \\
\hline UTI & 2 & 0.7 \\
\hline Viral fever & 64 & 23.7 \\
\hline Dengue/UTI & 1 & 0.4 \\
\hline Enteric fever with liver abscess & 1 & 0.4 \\
\hline Scrub typhus/P.vivax & 1 & 0.4 \\
\hline Scrub typhus/sepsis & 1 & 0.4 \\
\hline Scrub Typhus/Enteric & 1 & 0.4 \\
\hline Scrub Typhus/UTI & 1 & 0.4 \\
\hline Sepsis & 1 & 0.4 \\
\hline Tuberculosis & 1 & 0.4 \\
\hline Total & $\mathbf{2 7 0}$ & $\mathbf{1 0 0}$ \\
\hline
\end{tabular}

$38.1 \%$ (103) of the diagnosed patients were of Scrub Typhus aetiology, 23.7\% (64) of them were of miscellaneous aetiology, 15.9\% (43) of the cases were Dengue and Enteric fever accounted for 12.3\% (33). [Table 1]

\section{Discussion}

The purpose of this study was to identify and compare the various causes and clinical presentations of acute undifferentiated fever in a teaching hospital in Pondicherry. Majority of the patients were in the middle age group (mean age of 39.1) at risk for likely exposure to environmental pathogens. Mean duration of fever was 6.9 days and myalgia was the most common presenting feature with $71.5 \%$ patients followed by cough/dyspnoea with $39.6 \%$ and diarrhoea $10.4 \%$. The age group between 20 to 50 years is the economically productive period during which they have high chance of having contact with contaminated environment. The predominance in males is likely to be due to more chance of exposure to organisms due to their nature of work. Lower utilization of health care delivery facilities by females due to socio-cultural reasons could be another reason. Males were affected twice as that of females. This may be due to their easy exposure to mosquitoes and mites because of their outdoor activities.

The study revealed the heavy burden of tropical infections such as dengue, enteric fever, scrub typhus and malaria. Previous studies in Northern and Southern parts of the country have shown the similar results [3, $11,12,13]$. A similar study conducted by Singh R et al., from the region of Uttarakhand also showed that dengue, malaria, typhoid and enteric fever are the most 


\section{Original Research Article}

common aetiological agents of acute febrile illness [14]. Major causes of AUF identified were Scrub Typhus, Dengue fever, Enteric fever and undiagnosed fever taken to be that of viral aetiology. In India infectious disease remains the main cause of fever [15]. In a cohort study by Abrahamsen et al., from Southern India, bacterial infections (38\%) and TB (19\%) were the most common aetiological agents of fever [16].

Another study from east India reported that TB (53\%), neoplasms (17\%) and collagen vascular disorders (11\%) were the dominant causes. ${ }^{8}$ But our study diagnosed only 1 case of Tuberculosis which is partly due to a dedicated Tuberculosis and chest department functioning separately in our hospital.

In our study, Scrub Typhus was at the top of the list, constituting $38.1 \%$ of the infectious causes. In our study, we could establish the diagnosis in $76.3 \%$ of the cases.

Dengue, malaria, scrub typhus, enteric fever and leptospirosis have been identified as major causes of AUFI in Thailand, Malaysia and Nepal [17, 18, 19, 20].

Various studies conducted particularly in South India by Chrispal et al., Gopalakrishnan et al., and Kashinkunti et al., have also showed similar results [22, 22, 23]. In the miscellaneous group of our study, no definite cause was found in $23.7 \%$ (64) and was presumed to be of viral origin. Further studies are needed to identify the causes of fever in the miscellaneous group.

Second highest frequencies of diagnosed AUF were that of Dengue Fever, being diagnosed in 20\% (54) of our sampled population. These results underline the importance of awareness by health centre staff to be able to distinguish viral infections and be aware of their possible complications. The proportion of dengue fever among all fever cases has been estimated to be $14 \%$ in a population-based study in rural South India and $48 \%$ in a hospital based study in urban North India [24, 25].

Bleeding diathesis is a known feature of Dengue and Scrub typhus because of low platelet count and leakage from blood vessels. Bone marrow suppression, Immune mediated clearance, spontaneous aggregation of platelets to virus infected endothelium-all may be responsible for such thrombocytopenia. In our study, we found only 1 patient with bleeding episodes in the form of gum bleeding and/or melena who was diagnosed with Dengue. Therewere no death within our study population during the period of study.
Leptospirosis and Salmonella infections have been implicated in causation of one-third and one-tenth of all fever cases in 2 different studies [26, 27]. In our study group $12.3 \%$ (33) of the patients were diagnosed to have Enteric fever by Widal test and 1 case of Lepstospirosis by serology.

Despite AUF being common, the studies on its epidemiology remain limited. Recently, the public health system in India has initiated a more systematic integrated disease surveillance program (IDSP), which aims to compute the burden of infectious diseases, including AUFs, in a more comprehensive manner [28].

Malaria were less frequent in this study (5.2\%). All of them were of $P$. vivaxaetiology. UTI was a frequent diagnosis with 7 cases of $E$. coli, 1 case of Enterococci and 1 case of Staphylococci.

Mixed infection with more than one aetiological agent can result in an illness with overlapping symptoms, resulting in a situation where the diagnosis and management of such a patient could be challenging for the treating physician $[29,30,31,32,33]$. In our study there were 3 cases of Scrub Typhus with Dengue Fever and other isolated cases of Scrub Typhus with Malaria, Enteric Fever, UTI and Sepsis. There was also a case of Dengue with UTI. These cases were excluded from further analysis as they may show compounding features due to their mixed nature of disease. Symptoms of one disease may mimic with other disease which are also prevalent in this area. So, patients presenting with acute febrile illness should not be presumed to be suffering from single infection alone. The clinician should investigate thoroughly to look for other causes of fever.

In our study, the HIV infection was detected in only 1 patient despite the relatively high-risk study population (i.e., adults with febrile illness). This is important information given the general concern in about rising HIV infection rates in our country [34].

In spite of methodological difficulties, there was a large number of AUF that went undiagnosed. Rapid advances in molecular biology research and the availability of high throughput sequencers are a promising tool to reduce the number of undiagnosed AUF cases in the future. These advances may enable the identification of occult infections and previously unknown pathogens. This new method does not rely on a knowledge of the pathogens being sought but provides bulk sequencing of any nucleic acid present in the sample [35]. This 
technology to diagnose AUF should be further explored. We did not intend to perform an exhaustive search for all potential causes of fever among our febrile adults. Our main purpose was to describe the burden of some of the most likely pathogens, and to use this data as a foundation for further more focused research. Our results may underestimate the true burden for several reasons. First, hospital-based studies tend to underestimate disease incidence [36]. While this approach facilitates use of laboratory services, hospitalbased surveillance captures only the most severe illnesses in people who have access to hospital care. Second, antibiotics can be purchased without prescription in India. Undoubtedly, antibiotic use reduced the ability to isolate pathogens from blood cultures. This study demonstrates the usefulness of expanding microbiologic capacity in the developing world and the value of laboratory-based studies in sentinel institutions. Such studies provide a snapshot of important infections and can be periodically repeated for surveillance purposes. We have to be on the guard for emerging and re-emerging infections such as Dengue and Scrub Typhus.

Our study has a few limitations. We have focused on hospitalized adults (i.e., the most severely ill patients), our study findings should not be generalized to the acute febrile illness subtypes seen in outpatients or in the community. Patients were followed only until a diagnosis was confirmed or if patient recovered within the study period in case of miscellaneous fevers. Any patient who crossed over to FUO were not followed up. Treatment and recovery of the cases were not followed up after the diagnosis was made. Our results cannot be applied to infants and children. Several other infections that have been described to cause fever in the area were not systematically pursued. Important among this group are Japanese encephalitis, Hepatitis E, Epstein-Barrvirus, Hepatitis A, Hepatitis E, and Coxsackie virus, Influenza and other bacterial infections such as Q-fever, Brucellosisand Melioidosis

\section{Conclusion}

In conclusion, despite AUF being common, studies regarding its epidemiology are limited. In our study, serology is the most common diagnostic tool and the most prevalent aetiologies of AUF are Scrub Typhus, Dengue and Enteric fever. Our data showed that the etiologic spectrum of acute undifferentiated fever was widely distributed in our region. Scrub typhus was the major cause of acute undifferentiated fever next to Dengue and Enteric fever. There were a bulk of cases which were not diagnosed and put under miscellaneous aetiology of fever, this made the second largest group in our study next to the Scrub Typhus group. This bulk of cases are a reflection of how much more we need to improve our clinical and diagnostic methodologies in order to make a prompt and accurate diagnosis in spite of all the currently available methods.

The clinical evaluation of the subjects recruited for this study has not revealed any predictor symptoms or specific risk factors to differentiate between the different causes of AUF. The lack of a widely agreed upon definition of AUF makes comparison of patients with AUF between regions and countries difficult. Further studies need to be conducted using a standard definition of AUF by evaluating patients clinically and serologically for detecting infectious diseases.

\section{What this study contributes to present scenario?}

Our data showed that the etiologic spectrum of acute undifferentiated fever is widely distributed in our region. Scrub typhus was the major cause of acute undifferentiated fever next to Dengue and Enteric fever. This can guide us to concentrate our diagnostic process and treatment to focus on the more likely aetiologies from this region. There were a bulk of cases which were not diagnosed and put under miscellaneous aetiology of fever, this made the second largest group in our study next to the Scrub Typhus group. This bulk of cases are a reflection of how much more we need to improve our clinical and diagnostic methodologies in order to make a prompt and accurate diagnosis in spite of all the currently available methods.

\section{Funding: Nil, Conflict of interest: None Permission of IRB: Yes}

\section{Reference}

1. Susilawati TN, McBride WJ. Acute undifferentiated fever in Asia: a review of the literature. The Southeast Asian journal of tropical medicine and public health. 2014; 45 (3):719-26.

2. Manocha H, Ghoshal U, Singh SK, Kishore J, Ayyagari A. Frequency of leptospirosis in patients of acute febrile illness in Uttar Pradesh. The Journal of the Association of Physicians of India. 2004;52:623-5.

3. Joshi R, Colford Jr JM, Reingold AL, Kalantri S. Nonmalarial acute undifferentiated fever in a rural hospital in central India: diagnostic uncertainty and overtreatment with antimalarial agents. The American journal of tropical medicine and hygiene. 2008 Mar 1; 78 (3):393-9. 


\section{Original Research Article}

4. Crump JA. Time for a comprehensive approach to the syndrome of fever in the tropics. Transactions of the Royal Society of Tropical Medicine and Hygiene. 2014; 108 (2):61-2

5. Mueller TC, Siv S, Khim N, Kim S, Fleischmann E, Ariey F, et al. Acute undifferentiated febrile illness in rural Cambodia: a 3-year prospective observational study. Plo S one. 2014; 9 (4): e 958 68.

6. Mbonye AK, Lal S, Cundill B, Hansen KS, Clarke S, Magnussen P. Treatment of fevers prior to introducing rapid diagnostic tests for malaria in registered drug shops in Uganda. Malaria journal. 2013; 12: 131.

7. Phuong HL, de Vries PJ, Nagelkerke N, Giao PT, Hung le Q, Binh TQ, et al. Acute undifferentiated fever in BinhThuan province, Vietnam: imprecise clinical diagnosis and irrational pharmaco-therapy. Tropical medicine \& international health : TM \& IH. 2006; 11 (6): 869-79.

8. Reller ME, Wunder EA, Jr., Miles JJ, Flom JE, Mayorga O, Woods $\mathrm{CW}$, et al. Unsuspected leptospirosis is a cause of acute febrile illness in Nicaragua. PLoS neglected tropical diseases. 2014; 8 (7): e 2941.

9. Kumar V, Kumar V, Yadav AK, Iyengar S, Bhalla A, Sharma N, et al. Scrub typhus is an under-recognized cause of acute febrile illness with acute kidney injury in India. PLoS neglected tropical diseases. 2014;8 (1): e 2605.

10. Susilawati TN, McBride WJ. Undiagnosed undifferentiated fever in Far North Queensland, Australia: a retrospective study. International journal of infectious diseases : IJID : official publication of the International Society for Infectious Diseases. 2014; 27 : 59-64.

11. Thangarasu S, Natarajan $P$, Rajavelu $P$, Rajagopalan A, Devey JS. A protocol for the emergency department management of acute undifferentiated febrile illness in India. International journal of emergency medicine. 2011 Dec 1;4(1):57.

12. Kejariwal D, Sarkar N, Chakraborti SK, Agarwal V, Roy S. Pyrexia of unknown origin: a prospective study of 100 cases. Journal of postgraduate medicine. 2001 Apr 1; 47(2):104.
13. Singh R, Singh SP, Ahmad N. A study of etiological pattern in an epidemic of acute febrile illness during monsoon in a tertiary health care institute of Uttarakhand, India. Journal of clinical and diagnostic research: JCDR. 2014 Jun;8(6):MC01.

14. Jena B, Prasad M, Murthy S, Ramanarao G. Demand pattern of medical emergency services for infectious diseases in Andhra Pradesh-a geo-spatial temporal analysis of fever cases. India Emerg J. 2010 Jan;5:5-8.

15. Jung A, Singh MM, Jajoo U. Unexplained feveranalysis of 233 cases in a referral hospital. Indian journal of medical sciences. 1999 Dec;53(12): 535-44.

16. Abrahamsen SK, Haugen CN, Rupali P, Mathai D, Langeland N, Eide GE, Mørch K. Fever in the tropics: aetiology and case-fatality-a prospective observational study in a tertiary care hospital in South India. BMC infectious diseases. 2013 Jul 30;13(1):355.

17. Murdoch DR, Woods CW, Zimmerman MD, Dull PM, Belbase RH, Keenan AJ, Scott RM, Basnyat B, Archibald LK, Reller LB. The etiology of febrile illness in adults presenting to Patan hospital in Kathmandu, Nepal. The American journal of tropical medicine and hygiene. 2004 Jun 1;70(6):670-5.

18. Sripanidkulchai R, Lumbiganon P. Etiology of obscure fever in children at a university hospital in northeast Thailand. Southeast Asian journal of tropical medicine and public health. 2005 Sep 1; 36(5): 1243.

19. Leelarasamee A, Chupaprawan C, Chenchittikul M, Udompanthurat $\mathrm{S}$. Etiologies of acute undifferentiated febrile illness in Thailand. J Med Assoc Thai. 2004 May 1;87(5):464-72.

20. Ellis RD, Fukuda MM, McDaniel P, Welch K, Nisalak A, Murray CK, Gray MR, Uthaimongkol N, Buathong N, Sriwichai S, Phasuk R. Causes of fever in adults on the Thai-Myanmar border. The American journal of tropical medicine and hygiene. 2006 Jan 1;74(1):108-13.

21. Chrispal A, Boorugu H, Gopinath KG, Prakash JA, Chandy S, Abraham OC, Abraham AM, Thomas K. Scrub typhus: an unrecognized threat in South Indiaclinical profile and predictors of mortality. Tropical Doctor. 2010 Jul;40(3):129-33. 


\section{Original Research Article}

22. Gopalakrishnan S, Arumugam B, Kandasamy S, Rajendran S, Krishnan B, Balaji A. Acute undifferentiated febrile illness among adults-a hospital based observational study. J Evolut Med Dent Sci. 2013 Apr $8 ; 2$ (14):2305-19.

23. Kashinkunti MD, Gundikeri SK, Dhananjaya M. Acute undifferentiated febrile illness-clinical spectrum and outcome from a tertiary care teaching hospital of north Karnataka. Int J Biol Med Res. 2013; 4 (2): 3399-402.

24. Samuel PP, Thenmozhi V, Tyagi BK. A focal outbreak of dengue fever in a rural area of Tamil Nadu. The Indian journal of medical research. 2007 Feb; 125 (2): 179.

25. Thomas EA, John M, Bhatia A. Cutaneous manifestations of dengue viral infection in Punjab (north India). International journal of dermatology. 2007 Jul 1; 46 (7):715-9.

26. Lal M, Aggarwal A, Oberoi A. Seroprevalence of leptospirosis in patients of PUO in Ludhiana. Indian journal of pathology \& microbiology. 2007 Apr; 50 (2): 462-3.

27. Bhattacharya SS, Dash U. A sudden rise in occurrence of Salmonella paratyphi A infection in Rourkela, Orissa. Indian journal of medical microbiology. 2007 Jan 1;25(1):78.

28. Bachani D. Integration of disease surveillance in India: current scenario and future perspective. Indian journal of public health. 2006 Jan 1; 50 (1):7.

29. Pradutkanchana J, Pradutkanchana S, Kemapanmanus M, Wuthipum N, Silpapojakul K. The etiology of acute pyrexia of unknown origin in children after a flood.

30. Mushtaq MB, Qadri MI, Rashid A. Concurrent infection with dengue and malaria: an unusual presentation. Case reports in medicine. 2013 Mar 30; 2013.

31. Suresh V, Krishna V, Raju CH, Teja PS, Usha V. A rare case of triple infection with dengue, malaria and typhoid. Int J Res Dev Health. 2013;1(4):200-03.

32. Singhsilarak T, Phongtananant S, Jenjittikul M, Watt G. Possible acute coinfections in Thai malaria patients. Southeast Asian journal of tropical medicine and public health. 2006 Jan 1;37(1):1.

33. Sharma A, Raina R, Dhiman P, Bellad A, Madhabhavi I, Panda P. Rare coinfection of scrub typhus and malaria in immunocompetent person. Online Journal of Health and Allied Sciences. 2012 Apr 25; 11 (2 (12)).

34. Hawkes S, Santhya KG. Diverse realities: sexually transmitted infections and HIV in India. Sexually Transmitted Infections. 2002 Apr 1; 78 (suppl 1): i $31-9$.

35. Palacios R, Gazave E, Goñi J, Piedrafita G, Fernando O, Navarro A, Villoslada P. Allele-specific gene expression is widespread across the genome and biological processes. PLoS One. 2009 Jan 7; 4 (1): e4150.

36. Pappachan MJ, Sheela M, Aravindan KP. Relation of rainfall pattern and epidemic leptospirosis in the Indian state of Kerala. Journal of Epidemiology \& Community Health. 2004 Dec 1;58(12):1054-.

\section{How to cite this article?}

Uthaya Sankar M.K, Perumbadi S.L, Shenbagaram K, Hanushraj R. Clinical \& etiological profile of acute undifferentiated fever in patients admitted to a teaching hospital in Pondicherry. Int J Med Res Rev 2018;6(05):259-265. doi:10.17511/ijmrr. 2018.i05.04. 